\title{
Fitting the full SED of galaxies to put constraints on dust attenuation and star formation determinations
}

\author{
Veronique Buat, Elodie Giovannoli, Mederic Boquien and \\ Sébastien Heinis
}

Laboratoire d'Astrophysique de Marseille

Aix Marseille University and CNRS 38 rue Joliot Curie, 13013 Marseille, France

email: Veronique.Buat@oamp.fr

\begin{abstract}
The combination of far-IR and UV-optical rest-frame data has proved to be very efficient to extract physical parameters from the SEDs of galaxies. Using Herschel and ancillary data from the Herschel Reference Survey and GOODS-Herschel Key Projects, we show how dust attenuation properties can be estimated inside local galaxies as well as in the distant Universe.
\end{abstract}

Keywords. infrared: galaxies, dust: extinction, ultraviolet: galaxies

\section{Introduction}

The broad-band spectral energy distributions (SED) of galaxies are the combination of the emission from stars of all ages and interstellar dust which interact in a complex way through absorption and scattering. Stars emit from the UV to the near-IR whereas the mid- and far-IR emissions come from interstellar dust heated by the stellar emission. With the availability of mid- and far-IR data for large samples of galaxies, codes combining stellar and dust emissions to analyze SEDs are particularly useful to constrain dust attenuation and provide robust star formation rates. Herschel observations coupled to ancillary data provide us with UV-to-far-IR SEDs for galaxies from the local to the high redshift Universe. Here we use data from two key projects: the Herschel Reference Survey (Boselli et al. 2010) and the GOODS-Herschel project (Elbaz et al. 2011).

\section{CIGALE: a physically-motivated code}

We use the code CIGALE (Code Investigating GALaxy Emission) (http://cigale.oamp. fr) which provides physical informations about galaxies by fitting their UV-to-far-IR SED (Noll et al. 2009, Giovannoli et al. 2011). CIGALE combines a UV-optical stellar SED and an IR-emitting dust component. First, models are built, then each model is quantitatively compared to the observed SED, accounting for uncertainties in the observed fluxes. The probability function of each parameter is calculated and the estimated value of the parameter and its error correspond to the mean and standard deviation of this distribution. The robustness of the parameter estimations is checked with artificial catalogues constructed for each set of input data. Models are generated with a stellar population synthesis code, assuming a star formation history and a dust attenuation scenario as inputs. The star formation history implemented in CIGALE is the combination of two stellar components which mimic an old and a young stellar population. To model the 


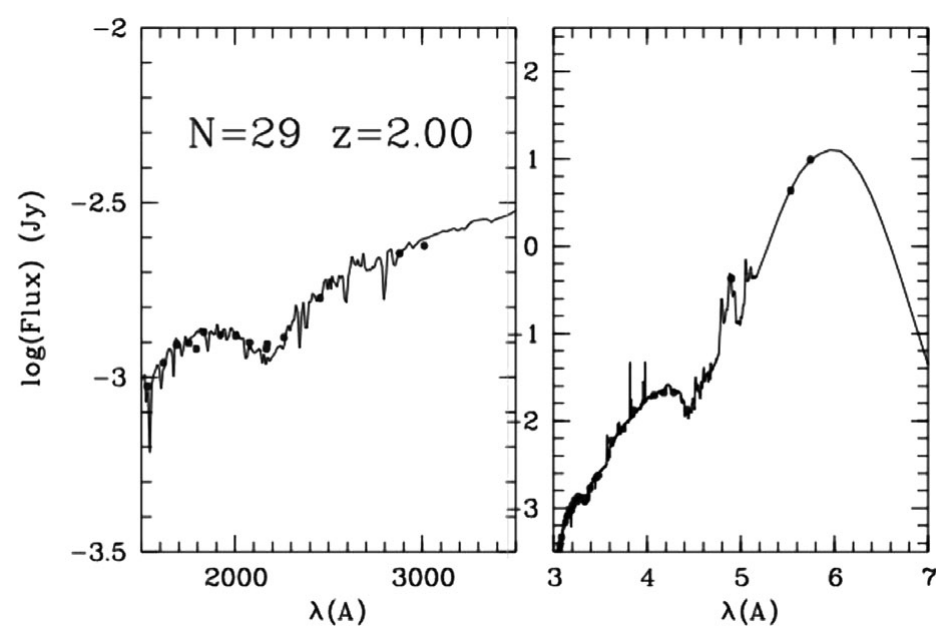

Figure 1. Example of SED fitting with CIGALE in the CDFS field for a galaxy at $z=2$. The whole SED is plotted in the right panel. The left panel is a zoom of the UV-optical range

attenuation by dust, the code uses the attenuation law of Calzetti et al. (2000) as a baseline, and offers the possibility of varying the steepness of this law and adding a bump centred at $2175 \stackrel{\circ}{A}$. We refer to Noll et al. (2009) for a complete description of the dust attenuation prescription. The energetic balance between dust-enshrouded stellar emission and re-emission in the IR is carefully conserved by combining the UV-optical and IR SEDs. The IR SEDs are built from the Dale \& Helou (2002) templates. CIGALE allows for additional dust emission from a dust-enshrouded AGN but does not include the unobscured emission of an active nucleus.

\section{Spatially resolved galaxies from the Herschel Reference Survey}

We exploit the spatial resolution of SPIRE maps to perform a local study of dust attenuation in a sub-sample of 7 galaxies extracted from the Herschel Reference Survey (Boselli et al. 2010). 11 photometric bands are used, FUV, NUV (GALEX), u, g, r, i, z (SDSS), h (2MASS), $70 \mu \mathrm{m}$ (SPITZER), 250 and $350 \mu \mathrm{m}$ (Herschel/SPIRE) are used. The method is illustrated in Figure 2 with HRS102 (Messier 99): the data are convolved and regridded to the same reference frame (at 350 microns) and a pixel-to-pixel analysis of the emission is performed by fitting the SED of each pixel with CIGALE. Maps are built for each output parameter of the code. Results of the SED fitting are used to study local variations of dust attenuation and their link to the UV emission (Boquien et al., in preparation).

\section{Dust attenuation curves in high redshift galaxies from the GOODS-Herschel survey}

Dust attenuation curves in external galaxies are useful for studying their dust properties as well as interpreting their intrinsic SEDs. These functions are not very well known in the UV range whether at low or high redshift. In particular the presence or absence of a UV bump at $2175 \AA$ remains an open issue that has consequences on the interpretation of broad band colours of galaxies involving the UV range. As part of the GOODS-Herschel Key Programme (Elbaz et al. 2011), the Herschel Space Observatory (Pilbratt et al. 2010) surveyed a small area of the Great Observatories Origins Deep Survey Southern 

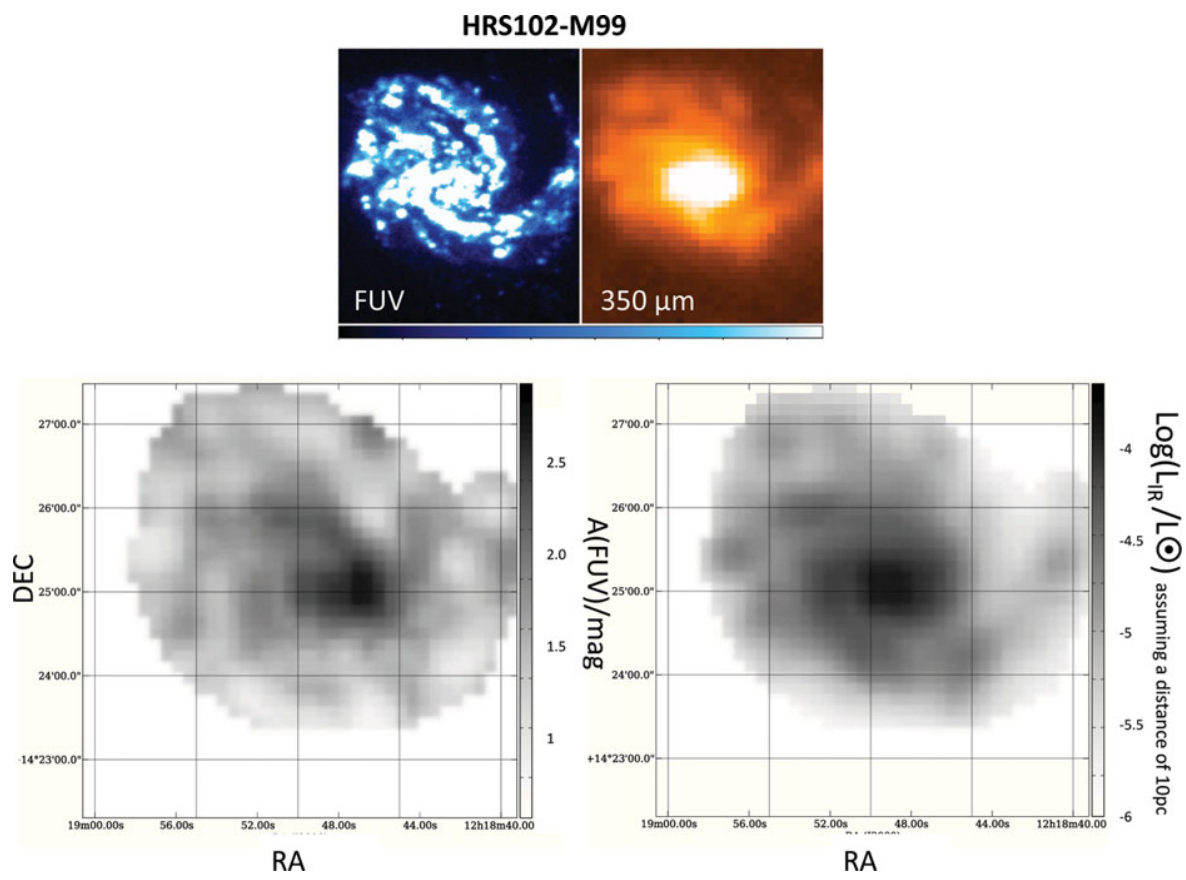

Figure 2. Application of CIGALE to HRS102

field (GOODS-S): $10^{\prime} \times 10^{\prime}$ centred on the CDFS were observed at 100 and $160 \mu \mathrm{m}$ over 264 hours by the PACS instrument (Poglitsch et al. 2010). Source extraction on the PACS images was performed at the prior position of Spitzer $24 \mu \mathrm{m}$ sources as described in Elbaz et al. (2011).

We use deep photometric data of the Chandra Deep Field South obtained with intermediate and broad band filters by the MUSYC project (Cardamone et al. (2010)) to sample the UV rest frame of galaxies with $1<z<2$. Herschel/PACS and Spitzer/MIPS data are used to measure the dust emission. We select 30 galaxies with high $\mathrm{S} / \mathrm{N}$ in all bands. Their SEDs from the UV to the far-IR are fitted using the CIGALE code and the characteristics of the dust attenuation curves obtained as outputs of the SED fitting process.

The average dust attenuation curve, shown in Fig. 3, is described well by a modified Calzetti et al. law slightly steeper than the original one and with a UV bump at $2175 \AA$ whose amplitude is $\sim 35 \%$ of the MW one. We propose an analytical expression of the average attenuation curve, which can be used to correct the SEDs of galaxies for dust attenuation.

$$
\frac{A(\lambda)}{A_{\mathrm{V}}}=\frac{k^{\prime}(\lambda)+D_{\lambda_{0}, \gamma, E_{\mathrm{b}}}(\lambda)}{4.05}\left(\frac{\lambda}{\lambda_{V}}\right)^{-0.13},
$$

where $\lambda_{V}=5500 \AA{ }^{\prime}, k^{\prime}(\lambda)$ is given in Calzetti et al. (2000) (Eq.4), and

$$
D_{\lambda_{0}, \gamma, E_{\mathrm{b}}}(\lambda)=\frac{1.26 \times 356^{2} \lambda^{2}}{\left(\lambda^{2}-2175^{2}\right)^{2}+\lambda^{2} \times 356^{2}} .
$$

The moderate amplitude of the bump, together with the substantial slope of our average attenuation curve in the UV, argues for a deficit of UV bump carriers in our sample galaxies as compared to the MW. For more details we refer to Buat et al. (2011). 


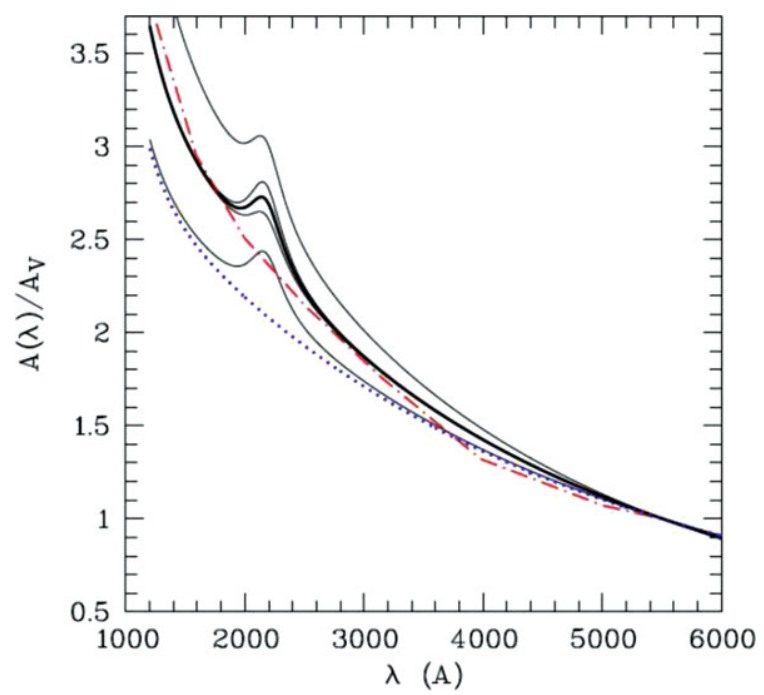

Figure 3. Average dust attenuation curve: thick line. The thin lines correspond to variations of $\pm 1 \sigma$ of the amplitude of the bump, and the slope of the continuum. The Calzetti et al. (2000) attenuation curve (dotted line) and the effective absorption curve of Charlot \& Fall (2000) for a starburst age of $310^{8}$ years (dot-dashed line) are shown in the figure for comparison.

\section{References}

Boselli, A. et al. 2010, PASP, 122, 261

Buat, V. et al. 2011, A\& A, 533, 93

Calzetti, D., Armus, L., Bohlin, R. C. et al. 2000, ApJ, 533, 682

Cardamone, C. N., van Dokkum, P. G., Urry, C. M. et al. 2010, ApJSS, 189, 270

Charlot, S. \& Fall, S. M. 2000, ApJ, 539, 718

Dale, D. A. \& Helou, G. 2002, ApJ, 576, 159

Elbaz, D. et al. 2011, A\&A, 533, 119

Giovannoli, E., Buat, V., Noll, S., Burgarella, D., \& Magnelli, B. 2011, A\&A, 525, 150

Noll S., Burgarella D., Giovannoli E. et al. 2009b, A\&A, 507, 1793

Pilbratt, G., Riedinger, J. R., Passvogel, T., et al. 2010, A\&A, 518, L1

Poglitsch, A., Waelkens, C., Geis, N. et al. 2010, A\&AA, 518, L2

\section{Discussion}

Clements: Naively, how many free parameters go into the CIGALE fits?

BuAT: The number of free parameters has to be fixed by the user. It clearly depends on the number of data available for each galaxy, for example one can fix the attenuation curve or use only one stellar component. Mock catalogues are very useful to decide which parameter can be free or have to be fixed.

Gallagher: Does CIGALE make linear combinations of templates, and if so are there issues in selecting templates to combine?

BUAT: In fact templates are based on a set of input parameters and built for each set of parameters. Templates are only pre-defined for the IR component. The set of parameters is chosen in order that the SED templates used for the fits reproduce the colours of the galaxies to be fitted. Therefore, CIGALE does not make a linear combination of templates. 